\title{
High Energy Physics Potential at Muon Colliders
}

Zohreh Parsa

Brookhaven National Laboratory

Physics Department

Upton, New York

April 2000 


\title{
HIGH ENERGY PHYSICS POTENTIAL AT MUON COLLIDERS
}

\author{
Z. Parsa* \\ Brookhaven National Laboratory, \\ Physics Department 510 A, Upton, NY 11973-5000, USA
}

\begin{abstract}
.
In this paper, high energy physics possibilities and future colliders are discussed. The $\mu^{+} \mu^{-}$collider and experiments with high intensity muon beams as the stepping phase towards building Higher Energy Muon Colliders (HEMC) are briefly reviewed and encouraged.
\end{abstract}

\section{INTRODUCTION}

The high energy physics community is interested in the potential of colliders beyond the $e^{+} e^{-}$colliders, and LHC, finding Higgs bosons (thus understanding the origin of electroweak symmetry breaking), and supesymmetric (SUSY) particles, etc. In devising a strategy for technical accelerator possibilities we can look at the historical record as represented by the Livingston Plot in Figure 1. There, accelerator energy is plotted as function of calendar time for various accelerators. It shows where we come from, where we are and where we are going.

Experiments over the last two decades have convincingly shown that the strong, electromagnetic, and weak forces are all closely related and are simply described by the "Standard Model." In particular the anticipated sixth quark, top, has been found at Fermilab, and the predicted properties of the $Z$ boson, one of the carriers of the weak force, have been tested to better than $0.1 \%$. Although there is now little doubt that the Standard Model is a very good description of the basic forces responsible for all atomic and nuclear physics, there remain many open questions $[1,2]$.

Perhaps the most urgent is to understand how masses of the elementary particles originate. To that end, new physics beyond what has been observed is required. The simplest possibility, the "Higgs Mechanism" predicts the existence of a fundamental Higgs Boson. Finding that elusive particle or whatever new physics is actually responsible for mass generation motivated the Superconducting Supercollider (SSC)

*) Supported by US Department of Energy contract DE-AC02-98CH10886

†)E-mail: parsa@bnl.gov 


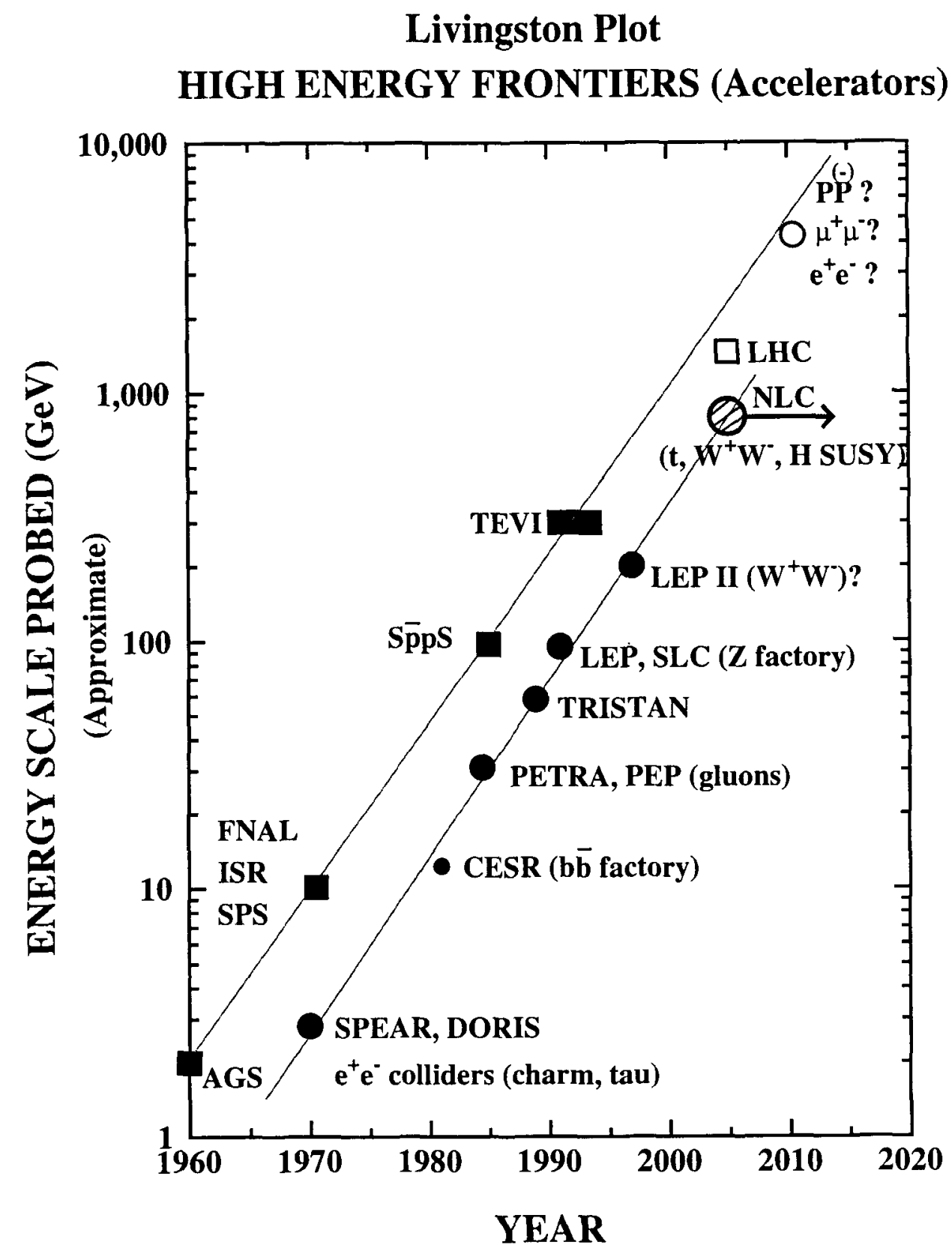

FIGURE 1. Livingston Plot - High Energy Frontiers (Accelerators) and their physics programs.

and remains the primary goal of the next generation of colliders. A number of other interesting and more elaborate models have been proposed, but there is as yet no direct experimental evidence supporting any of them.

Table 1 illustrates (a summary of the standard model), minimal spectrum of particles along with some of their basic properties. The fermions are grouped into three generations of spin $1 / 2$ leptons and quarks which span an enormous mass range. 


\section{FUTURE COLLIDERS - "NEW PHYSICS"}

Particle beam colliders are the primary tools for performing high energy physics research. Collisions of high energy particles produce events in which much of the energy of the beams can be converted into the masses of new heavy particles not normally found in nature. By studying the production and decay of these new particles, the underlying structure of the universe and the laws that govern it are unveiled.

High energy accelerators take us to new domains where top, Higgs, and "New Physics" can be directly produced and studied. The LHC, scheduled for 2005 will take us to $14 \mathrm{TeV}$ with very high luminosity $\simeq 10^{34} \mathrm{~cm}^{-2} \mathrm{~s}^{-1}$. Besides finding the Higgs, it will be capable of uncovering supersymmetry, $Z^{\prime}$ bosons, technicolor or many other scenarios with "new physics" $\lesssim 1 \mathrm{TeV}$. Beyond those facilities, new ideas and technologies are required. The Next Linear Collider $\left(e^{+} e^{-}\right)$offers an exciting viable possibility. The Recent, growing enthusiasm for a $\mu^{+} \mu^{-}$collider with high energy $\gtrsim 3 \mathrm{TeV}$ and luminosity $>10^{35} \mathrm{~cm}^{-2} \mathrm{~s}^{-1}$, if feasible, would be a significant technological leap forward.

High energy physicists are anxiously waiting for the next dramatic experimental discovery. Fortunately, anticipated future collider facilities offer broad discovery potential. The Fermilab main injector upgrade will allow the $p \bar{p}$ Tevatron to operate at $\sqrt{s} \lesssim 2 \mathrm{TeV}$ and luminosity $\sim 2 \times 10^{32}$. Those improvements broaden the discovery potential while allowing precision measurements and searches for rare $B$ and $\tau$ decays. The Higgs mass region of $110 \sim 130 \mathrm{GeV}$ may be explored via $W^{ \pm} H$ and $Z H$ associated production if the $H \rightarrow b \bar{b}$ mode is resolvable, [2] - [8].

Asymmetric $B$ factories provide new ways to explore CP violation. LEPII has achieved $e^{+} e^{-}$center-of-mass energy of about $202 \mathrm{GeV}$ and will push its energy to $\sqrt{s} \simeq 204 \mathrm{GeV}$ or higher. If a standard model or SUSY Higgs with mass $\lesssim 110$ $\mathrm{GeV}$ exists, it should be found. Perhaps, they will also get a first glimpse of SUSY. Furthermore, the $W^{ \pm}$mass has been measured to $\leq \pm 45 \mathrm{MeV}$ at LEPII, and Fermilab, providing an interesting constraint on the Higgs mass via quantum loop relations.

In some longer term $(\sim 2005)$, the LHC $p p$ collider with $\sqrt{s}=14 \mathrm{TeV}$ should find the Higgs scalar or tell us it doesn't exist. If SUSY exists $\lesssim 1 \mathrm{TeV}$, it will be discovered. Hopefully, completely unexpected revelations will also be made.

Beyond the LHC, various collider options are possible. The Next Linear Collider (NLC) would start $e^{+} e^{-}$collisions at $\sqrt{s}=500 \mathrm{GeV}$ and be upgradeable to 1$1.5 \mathrm{TeV}$. It would have high luminosity $>5 \times 10^{33}$ and polarization. The NLC also offers $\gamma \gamma, e^{-} e^{-}$, and $e^{-} \gamma$ collider options which expand its physics potential. There has been also some discussion of possible future $e^{+} e^{-}$colliders with $\sqrt{s} \simeq 5$ $\mathrm{TeV}$, a major step, if achievable. The NLC will be a superb tool for studying the Higgs, SUSY, Technicolor etc., $[9,10]$. Other possibilities include a $\mu^{+} \mu^{-}$collider and Very Large Hadron Collider ( $p p$ with $\sqrt{s} \simeq 100 \mathrm{TeV}$ or more) which are less advanced. The muon collider concept is very interesting, but require series studies and technology demonstrations. An effort at BNL will aim to produce very intense 
muon beams and use them to do physics (such as $\mu^{-} N \rightarrow e^{-} N$ ). Such hands on efforts combined with a vigorous $R \& D$ program could lead to the First Muon Collider (FMC). Various machine energies have been considered including $100 \mathrm{GeV}$, $500 \mathrm{GeV}, 3 \mathrm{TeV}$ etc., [14]- [19]. In the next section the concept of muon collider and parameter sets will be addressed. Recently, the concept of muon storage ring based Neutrino Source has generated considerable interest in the High Energy Physics community. Beside providing the first phase toward a muon collider, it would generate more intense and well collimated neutrino beams than currently available.

The Very Large Hadron Colidier(VLHC) with $\sqrt{s} \simeq 100 \mathrm{TeV}$ and $\mathcal{L} \simeq 10^{35}$ looks technically feasible but is very expensive. Does a Very Large Hadron Collider with $\sqrt{s} \simeq 100 \mathrm{TeV}$ have viability? Our SSC experience suggests a prohibitive cost and difficult construction issues because of its size. However, new ideas about inexpensive magnets and tunnels and/or a new technology could offer hope for the needed significant reduction in cost.

\section{MUON COLLIDER}

Figure 2 shows a schematic of a high energy muon collider components $[14,15]$. A high intensity proton source is bunch compressed and focused on a heavy metal target. The pions generated are captured by a high field solenoid and transferred to solenoidal decay channel within a low frequency linac. The linac reduces, by phase rotation the momentum spread of the pions and of the muons into which they decay. Subsequently, the muons are cooled by a sequence of ionization cooling stages, and must be rapidly accelerated to avoid decay. This can be done in recirculating accelerators (as at CEBAF) or in fast pulsed synchrotrons. Muon collisions occur in a separate high field collider storage ring with a single very low beta insertion.

A muon collider with center of mass energy less than about $10 \mathrm{TeV}$ can be circular and relative to NLC (a Next Linear Collider) of the same energy, it could be far smaller in size. For the same luminosity a muon collider can tolerate a far larger spot size than an electron linear collider since the muons make about 1000 crossings. Muon $\left(m_{\mu} / m_{e}=207\right)$ have the same advantage in energy reach as electron but has little beamstrahlung, thus very small energy spread is obtainable. In addition the direct coupling of lepton-lepton system to Higgs boson has a cross section proportional to the square of the lepton mass. thus the cross section for direct Higgs production from a $\mu^{+} \mu^{-}$collider is about 40,000 times that from an $e^{+} e^{-}$collider system.

A large effort has been devoted to design and assessing the feasibility of building a high energy muon collider at a $4-3 \mathrm{TeV}, .5-.4 \mathrm{TeV}$ and $.1 \mathrm{TeV}[14,15]$. Figure 2 illustrates concept of a $4 \mathrm{TeV}$ muon collider complex. Machines with energies higher than $3 \sim 4 \mathrm{TeV}$, have a significant beam current constraints from the neutrino radiation limits. Thus to reach the required high luminosities without unacceptable radiation hazards, a significant improvements in the muon emittance to the current base-line values are required. Although muon colliders remain a promising 


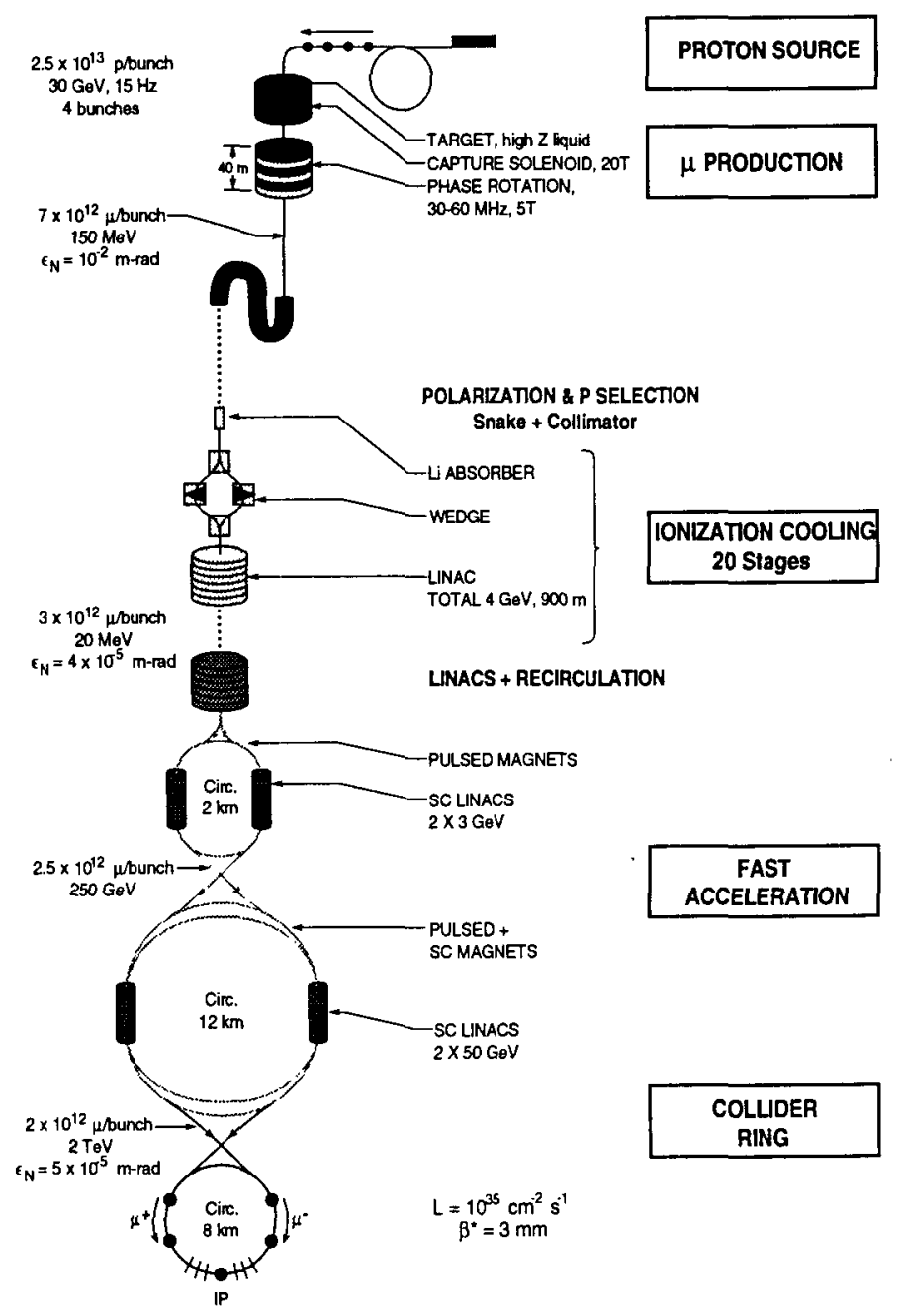

FIGURE 2. Schematic of a $4 \mathrm{TeV}$ Muon Collider.

complement, or alternative to $e^{+} e^{-}$colliders, much work is still needed, including demonstration of $\mu$ production and cooling, detector, and neutrino radiation.

A $100 \mathrm{TeV}$ muon collider provides a unique opportunity for exploring high energy physics, and mysteries of the elementary particles and their role in the universe. Table 2 [13] illustrates a 10 and $100 \mathrm{TeV}$ parameter sets distributed by workshop organizers. These parameters are speculative and require technical extrapolation much beyond present limits and may not be possible for many decades to come? Unfortunately, the estimates of the size, cost and radition problems seems to prohibit muon colliders at such energies, without some drastic new developments? For energies below $3 \mathrm{TeV}$, and for a fixed muon current, the neutrino radiation falls proportional to the energy cubed, which may be less of a problem? 


\section{PHYSICS POTENTIALS}

Muon Colliders have unique physics and technical advantages and disadvantages as compared to $e^{+} e^{-}$and hadron colliders and are to be considered as complementary. For the same energy and integrated luminosity, anything that can be done at $e^{+} e^{-}$, should be possible at $\mu^{+} \mu^{-}$collider, and more. E.g., possibilities for s-channel Higgs production, and a higher center-of mass energy with reduced backgrounds. Both of which are due to the large muon mass as compared to the electron mass. Higher energy may be crucial e.g., in improving signals for WW scattering, and the kinematical reach for pair production of SUSY particles.

The figure of merit in physics searches at an $e^{+} e^{-}$or $\mu^{+} \mu^{-}$collider, is expressed by the QED point cross section for $e^{+} e^{-} \rightarrow \mu^{+} \mu^{-}$:

$$
\sigma_{Q E D}(\sqrt{s})=\left(\frac{100[\mathrm{fb}]}{s[\mathrm{TeV}]}\right) \times\left(\frac{\alpha(s)}{\alpha\left(M_{z}^{2}\right)}\right)
$$

As before [16], we will neglect the factor $\left(\frac{\alpha(s)}{\alpha\left(M_{z}^{2}\right)}\right)$ as it varies slowly with s. Also, if the integrated luminosity needed for studying the new physics signals is:

$$
\left(\int L d t\right) \sigma_{Q E D} \gtrsim 1000 \text { events }
$$

then the $\mu^{+} \mu^{-}$collider design should be able to deliver an integrated luminosity of

$$
\left(\int L d t\right) \gtrsim 10 s\left[f b^{-1}\right] .
$$

If this is to be accumulated for one year of running time, the required luminosity estimate is

$$
L\left[\mathrm{fb}^{-1}\right] \gtrsim 10^{33} s\left[\mathrm{~cm}^{-2}\right]\left[\mathrm{sec}^{-1}\right] .
$$

E.g., The estimates of the luminosity requirements for the collider energies of interest (in parameter Table 2, distributed by organizers [13]) in this workshop are given below, (some of these energies and high luminosities may not be possible in practice?):

- For $\sqrt{s} \simeq 100[\mathrm{TeV}], L\left[\mathrm{fb}^{-1}\right] \gtrsim 10^{37}\left[\mathrm{~cm}^{-2}\right]\left[\mathrm{sec}^{-1}\right]$

- For $\sqrt{s} \simeq 10[\mathrm{TeV}], L\left[\mathrm{fb}^{-1}\right] \gtrsim 10^{35}\left[\mathrm{~cm}^{-2}\right]\left[\mathrm{sec}^{-1}\right]$

- For $\sqrt{s} \simeq 4[\mathrm{TeV}], L\left[\mathrm{fb}^{-1}\right] \gtrsim 10^{34}\left[\mathrm{~cm}^{-2}\right]\left[\mathrm{sec}^{-1}\right]$

- For $\sqrt{s} \simeq 3[\mathrm{TeV}], L\left[\mathrm{fb}^{-1}\right] \gtrsim 10^{33}\left[\mathrm{~cm}^{-2}\right]\left[\mathrm{sec}^{-1}\right]$

- For $\sqrt{s} \simeq 0.1[\mathrm{TeV}], L\left[\mathrm{fb}^{-1}\right] \gtrsim 10^{31}\left[\mathrm{~cm}^{-2}\right]\left[\mathrm{sec}^{-1}\right]$. 


\section{PHYSICS WITH INTENSE MUON BEAMS}

Using intense muon beams, forefront low energy research may be possible, including possibilities of: Precision measurements (e.g., muon decay $\tau_{\mu} \rightarrow G_{F}$, and Michel parameters); Neutrino source; Muon scattering; Muon capture $\mu^{-} p \rightarrow \nu_{\mu} n$. More interesting are the possibilities of Anomalus magnetic moment, Parity violation in muonic atoms (better than 1\%); T violation; and $\nu_{\mu}$ mass. Most interesting and compelling possibilities include the Muon number non-conservation - (rare or forbidden processes - Discovery would revolutionize physics.) such as

$$
\begin{aligned}
& \mu^{+} \rightarrow e \gamma ; \\
& \mu^{+} \rightarrow e^{+} e^{-} e^{+} ; \\
& \mu^{-} N \rightarrow e^{-} N .
\end{aligned}
$$

Other processes could include

$$
\begin{aligned}
& \mu^{-} N \rightarrow e^{+} N^{\prime} ; \\
& \mu^{-} N \rightarrow \mu^{+} N^{\prime} ; \\
& \mu^{+} e^{-} \rightarrow \mu^{-} e^{+} ; \\
& \mu^{-} e^{-} \rightarrow e^{-} e^{-} ; \\
& \mu^{+} e^{-} \rightarrow e^{+} e^{-} .
\end{aligned}
$$

\section{DISCUSSION}

The concept of muon collider once entirely speculative, now promises to extend the high energy frontier to an unprecedented domain, with center of mass energies of $3 \mathrm{TeV}$ or beyond as its goal. Considerable effort has already gone into the conceptual design of muon colliders, but much more work and study is needed. The muon Collider Collaboration represents a dedicated effort to address those issues and bring to realm the possibility of a future muon collider complex.

A $100 \mathrm{TeV}$ muon collider (energy of interest at this workshop) would provide a unique opportunity for exploring high energy physics, and mysteries of the elementary particles and their role in the universe. Unfortunately, the estimates of the size, cost and radiation problems seems to prohibit muon colliders at such energies, for many decades to come, without some drastic new technology developments?

Although a full high energy muon collider may take a considerable time to realize, intermediate steps in its direction are possible and could help facilitate the process. Employing an intense muon source to carry out forefront low energy research, such as the search for muon - number non - conservation, represents one interesting possibility. For example, the MECO proposal at BNL aims for $2 \times 10^{-17}$ sensitivity in their search for coherent muon - electron conversion in the field of a nucleus. 
To reach that goal requires the production, capture and stopping of muon at an unprecedented $10^{11} \frac{\mu}{\mathrm{sec}}$. If successful, such an effort would significantly advance the state of muon technology. More ambitious ideas for utilizing high intensity muon sources are also being explored. Indeed, if very high intensities, $\sim 10^{21} \frac{\nu}{\text { year }}$, are attained and nature has been kind in her neutrino mass and mixing parameters, one could envision a complete exploration of the $3 \times 3$ neutrino mixing matrix and even the detection of $\mathrm{CP}$ violation in the oscillation phenomena.

High intensity muon experiments, neutrino factories, and other intermediate steps toward the muon collider are extremely important. They will greatly expand our abilities and build confidence in the credibility of high energy muon colliders.

\section{REFERENCES}

1. Z. Parsa (Editor), Future High Energy Colliders AIP-Press CP 397 (1997).

2. W.J. Marciano, Keynote Address in Proc. of Snowmass 1996 and Proc. of Santa Barbara symposium on "Future High Energy Colliders" 1996 Ed. Z. Parsa, AIP CP 397, pp 11-25 (1997); ibid, private communication 1999.

3. C-N. Yang, Oskar Klein Memorial Lecture; Phys. Today 33,42 (1980); A. Zee, "Fearful Symmetry", Macmillan 1986.

4. S. Weinberg, Phys. Rev. Lett. 19 (1967) 1264; A. Salam, in Elementary Particle Theory, ed N. Svartholm (Almquist \& Wiksells, 1968) p. 367.

5. See A. Sirlin, Comments on Nucl. and Part. Phys., 21, 287 (1994).

6. D. Gross and F. Wilczek, Phys. Rev. Lett. 30, 1323 (1973); H. D. Politzer, Phys. Rev. Lett. 30, 1346 (1973).

7. W. Marciano, Phys. Rev. D29, 580 (1984).

8. A. Stange, W. Marciano, and S. Willenbrock, Phys. Rev. D50, 4491 (1994).

9. S. Kuhlman et al., Physics Goals of the Next Linear Collider, BNL report 63158.

10. ZDR Report for NLC, SLAC, May 1996; updates (1999).

11. See Physics working group summary and other papers in the HEMC'99 proceedings.

12. Proceedings of HEMC'99 "Studies on Colliders and Collider Physics at the Highest Energies: Muon Colliders at $10 \mathrm{TeV}$ and $100 \mathrm{TeV}$ ", 27 Sept.-1 Oct., 1999, Montauk, NY, USA.

13. B. King, Private comm.: provided a latex file for Table 2.

14. Muon Collider Collaboration; J. Norem private comm.: provided file for Fig. 2.

15. C.M. Ankenbrandt etal, Status of muon collider research and development and future plans, Phys. Rev. ST Accel. Beams 2, 081001 (1999), and references therein; $\mu^{+} \mu^{-}$ Collider, A Feasibility Study, BNL-52503, FERMILAB-Conf-96/092, LBNL-38946 (July 1996), Proceedings of the 1996 DPF Summer Study on High Energy Physics, Snowmass'96; Collaboration web page http://www.cap.bnl.gov/mumu.

16. V. Barger, M. Berger, K. Fujii, J. Gunion, T. Han, C. Heusch, W. Hong, S. Oh, Z. Parsa, S. Rajpoot, R. Thun, W. Willis, BNL-61593 (1995).

17. Z. Parsa, New High Intensity Muon sources and Flavor Changing Neutral Currents, World scientific Publishing, pp 147-153 (1998). 
TABLE 2. HEMC99 Parameter Sets For High Energy Muon Colliders

\begin{tabular}{|c|c|c|c|c|}
\hline $\begin{array}{l}\text { center of mass energy, } \mathrm{E}_{\mathrm{CoM}} \\
\text { additional description }\end{array}$ & $\begin{array}{c}0.1 \text { to } 3 \mathrm{TeV} \\
\text { MCC status report }\end{array}$ & $\begin{array}{c}10 \mathrm{TeV} \\
\text { evol. extrap. }\end{array}$ & $\begin{array}{c}100 \mathrm{TeV} \\
\text { evol. extrap. }\end{array}$ & $\begin{array}{c}100 \mathrm{TeV} \\
\text { ultracold beam }\end{array}$ \\
\hline 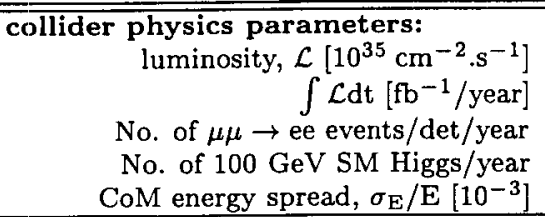 & $\begin{array}{c}8 \times 10^{-5} \rightarrow 0.5 \\
0.08 \rightarrow 540 \\
650 \rightarrow 10000 \\
4000 \rightarrow 600000 \\
0.02 \rightarrow 1.1 \\
\end{array}$ & $\begin{array}{l}10 \\
10000 \\
8700 \\
1.4 \times 10^{7} \\
0.42\end{array}$ & $\begin{array}{c}10 \\
10000 \\
87 \\
2.1 \times 10^{7} \\
0.080\end{array}$ & $\begin{array}{l}1000 \\
1.0 \times 10^{6} \\
8700 \\
2.1 \times 10^{9} \\
0.071 \\
\end{array}$ \\
\hline $\begin{array}{l}\text { collider ring parameters: } \\
\text { circumference, } \mathrm{C}[\mathrm{km}] \\
\text { ave. bending B field }[\mathrm{T}]\end{array}$ & $\begin{array}{c}0.35 \rightarrow 6.0 \\
3.0 \rightarrow 5.2\end{array}$ & $\begin{array}{l}15 \\
7.0\end{array}$ & $\begin{array}{r}100 \\
10.5\end{array}$ & $\begin{array}{r}100 \\
10.5\end{array}$ \\
\hline $\begin{array}{r}\text { beam parameters: } \\
\left(\mu^{-} \text {or }\right) \mu^{+} / \text {bunch, } \mathrm{N}_{0}\left[10^{12}\right] \\
\left(\mu^{-} \text {or }\right) \mu^{+} \text {bunch rep. rate, } \mathrm{f}_{\mathrm{b}}[\mathrm{Hz}] \\
6 \text {-dim. norm. emit., } \epsilon_{6 N}\left[10^{-12} \mathrm{~m}^{3}\right] \\
\epsilon_{6 N}\left[10^{-4} \mathrm{~m}^{3} \cdot \mathrm{MeV}^{3}\right] \\
\text { P.S. density, } \mathrm{N}_{0} / \epsilon_{6 N}\left[10^{22} \mathrm{~m}^{-3}\right] \\
\text { x,y emit. (unnorm.) }[\pi \cdot \mu \mathrm{m} . \mathrm{mrad}] \\
\mathrm{x}, \mathrm{y} \text { normalized emit. }[\pi \cdot \mathrm{mm} . \mathrm{mrad}] \\
\text { long. emittance }\left[10^{-3} \mathrm{eV} . \mathrm{s}\right] \\
\text { fract. mom. spread, } \delta\left[10^{-3}\right] \\
\text { relativistic } \gamma \text { factor, } \mathrm{E}_{\mu} / \mathrm{m}_{\mu} \\
\text { time to beam dump, } \mathrm{t}_{\mathrm{D}}\left[\gamma \tau_{\mu}\right] \\
\text { effective turns } / \mathrm{bunch} \\
\text { ave. current }[\mathrm{mA}] \\
\text { beam power }[\mathrm{MW}] \\
\text { synch. rad. critical } \mathrm{E}[\mathrm{MeV}] \\
\text { synch. rad. } \mathrm{E} \text { loss } / \mathrm{turn}[\mathrm{GeV}] \\
\text { synch. rad. power }[\mathrm{MW}] \\
\text { beam }+ \text { synch. power }[\mathrm{MW}] \\
\text { power density into magnet liner }[\mathrm{kW} / \mathrm{m}]\end{array}$ & $\begin{array}{c}2.0 \rightarrow 4.0 \\
15 \rightarrow 30 \\
170 \rightarrow 170 \\
2.0 \rightarrow 2.0 \\
1.2 \rightarrow 2.4 \\
3.5 \rightarrow 620 \\
50 \rightarrow 290 \\
0.81 \rightarrow 24 \\
0.030 \rightarrow 1.6 \\
473 \rightarrow 14200 \\
\text { no dump } \\
450 \rightarrow 780 \\
17 \rightarrow 30 \\
1.0 \rightarrow 29 \\
5 \times 10^{-7} \rightarrow 8 \times 10^{-4} \\
7 \times 10^{-9} \rightarrow 3 \times 10^{-4} \\
1 \times 10^{-7} \rightarrow 0.010 \\
1.0 \rightarrow 29 \\
1.0 \rightarrow 1.7\end{array}$ & $\begin{array}{c}3.0 \\
27 \\
85 \\
1.0 \\
3.5 \\
0.81 \\
38 \\
21 \\
0.60 \\
47300 \\
\text { no dump } \\
\text { i040 } \\
55 \\
131 \\
0.012 \\
0.017 \\
0.91 \\
130 \\
4.3\end{array}$ & $\begin{array}{c}0.80 \\
7.9 \\
10 \\
0.12 \\
8.0 \\
0.018 \\
8.7 \\
47 \\
0.113 \\
473000 \\
1.0 \\
1350 \\
4.0 \\
100 \\
1.75 \\
25 \\
99 \\
200 \\
1.2\end{array}$ & $\begin{array}{c}0.19 \\
65 \\
1.0 \times 10^{-3} \\
1.2 \times 10^{-5} \\
19000 \\
4.4 \times 10^{-4} \\
0.21 \\
8.1 \\
0.100 \\
473000 \\
1.0 \\
1350 \\
7.8 \\
198 \\
1.75 \\
25 \\
195 \\
390 \\
2.4\end{array}$ \\
\hline $\begin{array}{r}\text { interaction point parameters: } \\
\text { spot size, } \sigma_{x, y}[\mu \mathrm{m}] \\
\text { bunch length, } \sigma_{z}[\mathrm{~mm}] \\
\beta_{x, y}^{*}[\mathrm{~mm}] \\
\text { ang. divergence, } \sigma_{\theta}[\mathrm{mrad}] \\
\text { ip compensation factor: } \mathrm{N}_{0} / \mathrm{N}_{0, \mathrm{eff}} \\
\text { beam-beam tune disruption, } \Delta \nu \\
\text { pinch enhancement factor, } \mathrm{H}_{\mathrm{B}} \\
\text { beamstrahlung frac. E loss/collision }\end{array}$ & $\begin{array}{c}3.3 \rightarrow 290 \\
3.0 \rightarrow 140 \\
3.0 \rightarrow 140 \\
1.1 \rightarrow 2.1 \\
1 \\
0.015 \rightarrow 0.051 \\
1.00 \rightarrow 1.01 \\
\text { negligible }\end{array}$ & $\begin{array}{c}1.3 \\
2.2 \\
2.1 \\
0.63 \\
1 \\
0.085 \\
1.08 \\
6.8 \times 10^{-8}\end{array}$ & $\begin{array}{c}0.21 \\
2.5 \\
2.5 \\
0.086 \\
1 \\
0.100 \\
1.11 \\
1.5 \times 10^{-6}\end{array}$ & $\begin{array}{c}0.015 \\
0.49 \\
0.49 \\
0.030 \\
10 \\
0.100 \\
1.11 \\
9.0 \times 10^{-7}\end{array}$ \\
\hline $\begin{array}{r}\text { final focus lattice parameters: } \\
\text { max. poletip field of quads., } \mathrm{B}_{5 \sigma}[\mathrm{T}] \\
\text { max. full aper. of quad., } \mathrm{A}_{ \pm 5 \sigma}[\mathrm{cm}] \\
\text { quad. gradient, } 2 \mathrm{~B}_{5 \sigma} / \mathrm{A}_{ \pm 5 \sigma}[\mathrm{T} / \mathrm{m}] \\
\beta_{\max }[\mathrm{km}] \\
\text { ff demag., } M \equiv \sqrt{\beta_{\max } / \beta^{*}} \\
\text { chrom. quality factor, } Q \equiv M \cdot \delta\end{array}$ & $\begin{aligned} 6 & \rightarrow 12 \\
14 & \rightarrow 24 \\
50 & \rightarrow 90 \\
1.5 & \rightarrow 150 \\
220 & \rightarrow 7100 \\
0.007 & \rightarrow 11\end{aligned}$ & $\begin{array}{c}15 \\
22 \\
140 \\
580 \\
17000 \\
10\end{array}$ & $\begin{array}{c}20 \\
19 \\
210 \\
19000 \\
89000 \\
10\end{array}$ & $\begin{array}{c}20 \\
6.6 \\
610 \\
64000\end{array}$ \\
\hline $\begin{array}{r}\text { neutrino radiation parameters: } \\
\text { collider reference depth, } \mathrm{D}[\mathrm{m}] \\
\text { ave. rad. dose in plane }[\mathrm{mSv} / \mathrm{yr}] \\
\text { str. sec. len. for } 10 x \text { ave. rad. }[\mathrm{m}] \\
\nu \text { beam distance to surface }[\mathrm{km}] \\
\nu \text { beam radius at surface }[\mathrm{m}]\end{array}$ & $\begin{array}{c}10 \rightarrow 300 \\
3 \times 10^{-5} \rightarrow 0.03 \\
1.3 \rightarrow 2.2 \\
11 \rightarrow 62 \\
4.4 \rightarrow 24\end{array}$ & $\begin{array}{l}100 \\
3.8 \\
1.0 \\
36 \\
0.8\end{array}$ & $\begin{array}{c}100 \\
2.5 \\
4.2 \\
36 \\
0.08\end{array}$ & $\begin{array}{c}100 \\
4.9 \\
4.2 \\
36 \\
0.08\end{array}$ \\
\hline
\end{tabular}

18. Z. Parsa, Polarization and Luminosity requirements for the First Muon Collider, in AIP Conf. Proc. 472, pp. 251-259, (1998).

19. Z. Parsa, Muon Storage Rings - Neutrino Factories, in Proceedings of NNN'99, SUNY Stony Brook, NY. AIP-Press (2000); References therein. 